\title{
Complex population dynamics as a competition between multiple time-scale phenomena
}

\author{
Ioana Bena* and Michel Drot \\ University of Geneva, Theoretical Physics Department, \\ Quai E. Ansermet no. 24, 1211 Geneva 4, Switzerland \\ Janusz Szwabińsk: \\ University of Geneva, Theoretical Physics Department, \\ Quai E. Ansermet no. 24, 1211 Geneva 4, Switzerland \\ and Institute of Theoretical Physics, University of Wroctaw, Pl. M. Borna 9, 50-204 Wroctaw, Poland \\ Andrzej Pȩkalsk \\ Institute of Theoretical Physics, University of Wroctaw, Pl. M. Borna 9, 50-204 Wroctaw, Poland
}

(Dated: October 30, 2018)

\begin{abstract}
The role of the selection pressure and mutation amplitude on the behavior of a single-species population evolving on a two-dimensional lattice, in a periodically changing environment, is studied both analytically and numerically. The mean-field level of description allows to highlight the delicate interplay between the different time-scale processes in the resulting complex dynamics of the system. We clarify the influence of the amplitude and period of the environmental changes on the critical value of the selection pressure corresponding to a phase-transition "extinct-alive" of the population. However, the intrinsic stochasticity and the dynamically-built in correlations among the individuals, as well as the role of the mutation-induced variety in population's evolution are not appropriately accounted for. A more refined level of description, which is an individual-based one, has to be considered. The inherent fluctuations do not destroy the phase transition "extinct-alive", and the mutation amplitude is strongly influencing the value of the critical selection pressure. The phase diagram in the plane of the population's parameters - selection and mutation is discussed as a function of the environmental variation characteristics. The differences between a smooth variation of the environment and an abrupt, catastrophic change are also addressesd.
\end{abstract}

PACS numbers: 87.10.+e, 0.2.70.Lq

\section{INTRODUCTION}

The study of population dynamics is a recurrent subject of interdisciplinary research ever since the pioneering works of Lotka [1] and Volterra [2]. Most of the situations involve interacting multi-agent systems, that evolve in a spatially-extended environment, and exhibit complex cooperative behavior, see e.g. [3, 4]. Such systems are generally far from equilibrium and highly nonlinear. Moreover, it has been recognized that simple mean-field (MF) like approaches are not always able to grasp all the richness of the behavior of such systems [5]. The stochastic aspect of the dynamics, as well as the use of discrete spatial and/or temporal variables to characterize the population evolution may play an important role [6, 7]. Also, the inherent discrete nature of a population, i.e., the fact that it consists of a finite, integer number of individuals, was found to be sometimes an influencial factor of the dynamics, see [8, 9].

Therefore, the methods developed in nonequilibrium

\footnotetext{
*Electronic address: ioana.bena@physics.unige.ch

${ }^{\dagger}$ Electronic address: michel.droz@physics.unige.ch

${ }^{\ddagger}$ Electronic address: janusz.szwabinski@physics.unige.ch

$\S$ Electronic address: apekal@ift.uni.wroc.pl
}

statistical physics are particularly well-suited for the study of such systems. In particular, the so-called individual-based model (IBM) approach is able to account for all the above-cited ingredients of the dynamics.

A major part of the existing literature is investigating various evolution mechanisms in a constant environment, like, e.g., Darwinian selection leading to the elimination of the ill-adapted individuals [10], genetic heritage and accumulation of diseases [11], mutations [12], development of hunting/avoiding strategies of predators/preys [13], topology of the network connecting the individuals [14], etc. As a result of the nonlinear stochastic dynamics associated with the above mechanisms, nontrivial collective properties are emerging. In particular, depending on the values of the control parameters characterizing a system, different steady states are possible 15] and a phase diagram can be drawn.

However, a fundamental question concerning the dynamics of populations or ecosystems is their robustness with respect to external perturbations, like, for example, a change in their environment. Such an environmental change can be abrupt (due, e.g., to some catastrophic events) or rather smooth and periodic (due, e.g., to slow climatic modifications). Although the climatic changes recorded in paleoclimatic data [16] could hardly be called perfect oscillations, one notices a certain degree of periodicity. Periodic changes of the habitat are also assumed 
in most of the studies done both by biologists and physicists, see e.g. [17, 18, 19], and can be regarded as a rather satisfactory approximation to the real data, as far as extracting essential qualitative features is concerned.

One expects on general grounds that new complex behavior of the populations will emerge due to the competition between the characteristic intrinsic time scales and nonlinearities of the system and the time scale of the environmental perturbation. This is an aspect that, to our knowledge, has not been investigated systematically in the existing literature. Indeed, most of the previous studies on related points (see, e.g., [5, 20, 21]) are only reporting on the observed complex dynamics, without further analysis of its rooting. In this paper we shall thus address the role of selection pressure and mutation amplitude on the relevant properties of a single-species population which lives in a periodically changing habitat. Their interplay with the magnitude and period of the environmental changes will be a fundamental point of the analysis.

The paper is organized as follows: in the following section we present the details of the model; its implementation, both in a MF-like and an IBM-type of approach, are described in Sec. III. Sec. IV presents the analytical and numerical results obtained in the MF approximation. Section V resumes the main results of the IBM simulations, and compares them to the results of the MF approach. Finally, conclusions and perspectives are relegated to Sec. VI.

\section{THE MODEL}

We consider a population that consists of hermaphrodite individuals living in a spatially-extended habitat, which is represented here as a two-dimensional square lattice of size $L \times L$. We assume non-permeable boundary conditions, meaning that the individuals cannot cross the borders of the lattice. Moreover, the lattice has a finite carrying capacity, which comes from an exclusion assumption, according to which there is at most one individual in each lattice node.

The dynamics of the population is the result of several elements, namely natural selection, individual motion, mating and reproduction, whose modeling is described below.

A. Natural selection. Individual trait, optimum, fitness, selection pressure, extinction probability.

Each individual is characterized by its trait, or phenotype, which may correspond to various specific properties, such as e.g. the metabolic rate, the body size, or may encode a rather complex ensemble of properties. The trait of the " $i$-th" individual is represented here, for simplicity, through a real number $z_{i} \in[0,1]$. This number is fixed at the individual's birth, and remains constant. This simplified way to characterize an individual has been often used both by biologists [20, 21] and by physicists 25]. Its comparison with more elaborated descriptions, that take into account further details of the genetic structure of the individuals, was addressed, for example, in [24].

The population lives in an environment whose influence on the individuals is encoded in the value of another number $\varphi \in[0,1]$, the so-called optimum. The optimum is that value of the trait which guarantees for its owner maximum chance of survival. In principle, the optimum could be spatial and/or time-dependent. In this work the optimum has the same value in all the sites of the lattice, however its value can vary in time.

The degree of agreement of the individual trait $z_{i}$ with the optimum determines the individual extinction probability per unit time (or extinction rate),

$$
p_{i}=p_{0}\left[1-\exp \left(-\frac{\mathcal{S}}{f_{i}}\right)\right]
$$

where $\mathcal{S}$ is a parameter which models the selection pressure (SP) of the environment and constitutes a main control parameter of the system. The fitness $f_{i}$ of the individual $i$ is defined as

$$
f_{i}=1-\left|z_{i}-\varphi\right|
$$

A perfectly-adapted individual has a fitness equal to 1, and thus a minimum possible extinction rate. A fitness smaller than 1 corresponds to a worse adaptation; $f_{i}$ goes to zero for the completely unadapted individuals, that have the maximum possible extinction rate, equal to $p_{0}$. A variation in time of the fitness of one individual is resulting only from the variation of the optimum.

The constant $p_{0}$ in the definition (1) of the extinction rate is related to the choice of the unit time and it depends on whether we are considering the continuoustime MF-type model, or the discrete-time IBM-type of approach, see below.

The choice we made of the extinction rate (1), the implicit definition of the SP parameter $\mathcal{S}$ and that of the fitness (21) are frequently encountered in the biological literature, see e.g. 21]. Other choices, and thus other ways of measuring the "selection pressure" and the "individual fitness", are possible. However, most of them can be mapped one onto the other and/or account for equivalent qualitative aspects of the interaction between the individuals and their environment.

\section{B. Individual motion.}

If an individual survives, it can move to its surroundings. The simplest possibility, that we shall adopt hereafter, is a random-walk, diffusive-like motion. For example, in the discrete-time IBM-type of approach, in one time step the individual jumps on the lattice, from its initial location to a randomly chosen nearest-neighbor one (i.e., a site within the von Neumann neighborhood of the initial node), provided that the chosen site is empty, and that it lies within the boundaries of the system. If none 
of the four first-neighbor nodes is empty, then the individual cannot move, and thus cannot mate (see below).

\section{Mating and reproduction. Heredity and mutation.}

Suppose an individual $i$ reaches a destination node. Then, if there are other individuals ("neighbors") in the nearest-neighborhood of this destination site, the individual "i" choses at random one of these neighbors (call it " $j$ ") for mating [35]. The pair of individuals $i$ and $j$ may afterwards give birth to offsprings, whose number cannot exceed a prescribed value $N_{\text {off }}$. At their birth, the progenies are placed, at random, on the empty nodes of the joint nearest-neighborhoods of the two parents (that counts 6 sites); therefore, the maximum number of offsprings $N_{\text {off }} \leqslant 6$. If there is no room in this neighborhood for putting an offspring, then this one is not born.

The trait of a progeny $k$ coming from parents $i$ and $j$ is determined by the parents' traits (heredity), but it can also present some "variations" due to different random factors, such as recombination, mutations, etc. We shall consider

$$
z_{k}=\frac{1}{2}\left(z_{i}+z_{j}\right)+m_{k},
$$

where $m_{k}$ represents these variations. It brings diversification into the phenotypic pool of the population and we call it conventionally mutation. For simplicity, we shall admit that $m_{k}$ is a random number, uniformly distributed in the interval $[-\mathcal{M}, \mathcal{M}]$, where $0<\mathcal{M}<1$ is called hereafter the mutation amplitude (MA) and is a control parameter of the system [36]. Moreover, if Eq. (3) leads to $z_{k}>1$ or $z_{k}<0$, then one renormalizes it by resetting it to $z_{k}=1,0$, respectively. This means simply that the trait of the individuals cannot overcome some fixed limits. This choice (3) for the trait of an offspring is often made in the biological literature [21].

The population dynamics is thus driven by two main "forces" that are acting, to some extent, in opposite directions: selection and mutation, characterized, respectively, through the values of the control parameters $\mathcal{S}$ and $\mathcal{M}$. Selection, combined with heredity, tries to bring the average trait close to the instantaneous optimum, while mutation introduces diversity in the individual traits, and thus is broadening the distribution of the population's traits.

\section{IMPLEMENTATIONS OF THE MODEL}

The study of this system can be made on different levels of modelling. The simplest one, which has the advantage to allow, up to a certain extent, for analytic investigations, is a MF-like level. The drawback of this simple approach is to neglect fluctuations, which can play a crucial role. We are therefore proposing an investigation based on IBM-type simulations, for which the fluctuations are naturally built-in. A comparison of the results of these two approaches is then made.

\section{A. Mean-field like approach}

The main quantity of interest in this approach, that neglects the possible correlations between lattice sites, is the average population number density $c(t)$. One writes down a continuous-time evolution equation for $c(t)$ by taking into account the different dynamical processes described above,

$$
\frac{d c(t)}{d t}=c\left[R_{1}(c) R_{2}(c)-p\right] .
$$

This represents the balance between births, described by the nonlinear term $\left[c R_{1}(c) R_{2}(c)\right]$, and deaths, corresponding to the $(-c p)$ term. The meaning of these terms is as following:

(a) $p$ is the mean extinction probability per unit time, and, as resulting from Eqs. (1), (2), it reads

$$
p=p_{0}\left[1-\exp \left(-\frac{\mathcal{S}}{1-|\varphi-\langle z\rangle|}\right)\right],
$$

where $\langle z\rangle$ is the mean value of the trait. The time scale will be chosen such that the constant $p_{0}=1$.

In agreement with Eq. (3), the MF approach does not allow for a self-consistent evaluation of the mean trait $\langle z\rangle$; it remains thus an arbitrary constant $\in[0,1]$. The role of the mutation is practically eliminated in the MF description, and the only remaining control parameter is the selection $\mathcal{S}$.

(b) $R_{1}(c)$ expresses the probability that an individual jumps, per unit time, to a randomly-chosen empty nearest-neighbor site,

$$
R_{1}(c)=r_{0}\left(1-c^{4}\right),
$$

where $\left(1-c^{4}\right)$ is simply the probability that at least one of these four neighbor sites is empty. The constant $r_{0}$ depends on the choice of the unit time. If the unit time is fixed by chosing $p_{0}=1$, then $r_{0}$ is also fixed, and it measures the ratio between the mean survival time of an individual and its characteristic diffusion time on the lattice.

(c) The term $R_{2}(c)$ describes the probability that the considered individual encounters at least one nearestneighbor at the destination site and it produces a certain number of offsprings according to the model rules:

$$
R_{2}(c)=N_{\text {off }} c(1-c)^{N_{\text {off }}} .
$$

One is supposing implicitely that the time scale related to mating and reproduction is much shorter than both the time scales of survival and diffusion of an individual.

It can be easily shown that $r_{0} \gg 1$ implies a rapid increase of $c$ till the stationary saturation value $c=1$, while $r_{0} \ll 1$ leads to an extinction of the population. In the foregoing we shall thus focus on the case when diffusion and extinction have comparable characteristic time scales, and we shall fix throughout $r_{0}=1$. 
One is then led to the following MF equation:

$$
\begin{aligned}
\frac{d c(t)}{d t} & =N_{\text {off }} c^{2}\left(1-c^{4}\right)(1-c)^{N_{\text {off }}} \\
& -c\left[1-\exp \left(-\frac{\mathcal{S}}{1-|\varphi-\langle z\rangle|}\right)\right] .
\end{aligned}
$$

This one can be mapped onto the equation of motion of an overdamped particle in a potential $U_{\text {sel }}(c)$ whose actual profile depends on the selection parameter $\mathcal{S}$,

$$
\frac{d c(t)}{d t}=-\frac{d U_{\mathrm{sel}}(c)}{d c}
$$

This point of view will prove to be very useful in the discussion of the results presented in Sec. IV]

\section{B. IBM-type simulations}

The IBM simulation algorithm considers the individuals distributed on the lattice nodes, the initial condition being represented by their positions and the prescribed values of the individual traits. Different initial conditions were considered, see Sec. $\mathrm{V}$ below. The individuals are evolving, at discrete Monte-Carlo time steps (MCS, defined hereafter), according to the stages $A-C$ of the dynamics as described in the Introduction, namely:

$A$. At a given time $t$ an individual $i$ is picked at random, and its extinction probability $p_{i}$, corresponding to one MCS, is determined according to Eqs. (11), (2). The constant $p_{0}$ in Eq. (1) is chosen equal to 1 . Then a random number $r$ is extracted from an uniform distribution in the range $[0,1]$; if $r<p_{i}$, the individual dies, otherwise it survives.

$B$. If it survives, the individual $i$ jumps at random to one of the empty nearest-neighbor nodes on the lattice.

$C$. Then it possibly mates and produces offsprings.

If at the time $t$ there are $N(t)$ individuals in the system, then the above steps $A-C$ are repeated $N(t)$ times; this constitutes one MCS, the unit-time of the simulations. Finally, the time is advanced by one step, $t \rightarrow t+1$, and the above algorithm is repeated.

In the next two Sections we shall present and then compare the results obtained at each of these two levels of modeling.

\section{MEAN-FIELD TYPE ANALYSIS}

\section{A. Constant environment}

Consider first the case of a habitat with constant optimum $\varphi=0.5$, and a population described by a mean trait $\langle z\rangle=0.5$ [37]. We shall suppose throughout that $N_{\text {off }}=6$.

One can use simple arguments of the theory of deterministic dynamical systems to describe the evolution of the mean population concentration $c(t)$. The stationary

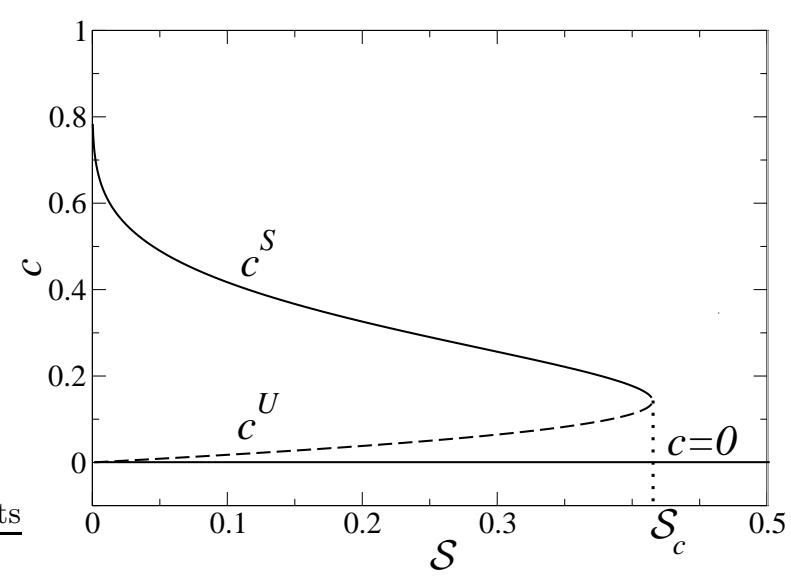

FIG. 1: MF stationary solutions for the concentration $c$ as functions of the selection parameter $\mathcal{S}$. Continuous lines represent the stable solutions $c=c^{S}$ and $c=0$, and the dashed line represents the unstable solution $c=c^{U}$. At $\mathcal{S}=\mathcal{S}_{c} \approx 0.417$ the stable $c^{S}$ and unstable $c^{U}$ solutions disappear, and for $\mathcal{S}>\mathcal{S}_{c}$ the only remaining stationary solution is the absorbing state $c=0$. The other parameters are $\varphi=\langle z\rangle=0.5$, and $N_{\text {off }}=6$.

concentrations as functions of the unique control parameter $\mathcal{S}$ are represented in Fig. 1. At a critical value of the selection $\mathcal{S}=\mathcal{S}_{c} \approx 0.417$ a discontinuous phase transition takes place in the system. For $\mathcal{S}<\mathcal{S}_{c}$, the system presents two stable states, namely $c^{S} \neq 0$ and the absorbing state $c=0$, and their respective basins of attraction are separated by the unstable solution $c^{U}$. At $\mathcal{S}=\mathcal{S}_{c}$ the stable $c^{S}$ and unstable $c^{U}$ critical points of the overdamped dynamics (4) disappear. For large $\mathcal{S}>\mathcal{S}_{c}$ the population gets extinct in the long-time limit, no matter what was its initial concentration $c(0)$.

The standard linear stability analysis 26] allows also to determine the characteristic times for the linear relaxation to the stable point $c^{S}$, (denoted $T_{\text {rel }}^{S}$ ) and, for the escape from the immediate vicinity of the unstable point $c^{U}$, denoted by $T_{\mathrm{esc}}^{U}$. One can also get the analytic expression for the linear relaxation time to the absorbing state $c=0$, namely $T_{\text {rel }}^{0}=[1-\exp (-\mathcal{S})]^{-1}$. These characteristic times offer an indication on the order of magnitude of the nonlinear relaxation times that correspond to the fully nonlinear dynamics (9). Moreover, as shown numerically, for a wide domain of intermediate values of the selecion pressure $0<\mathcal{S}<\mathcal{S}_{c}$, they are practically insensitive to changes in $\mathcal{S}$. This key aspect is implicitely taken into account in discussing the cases (i) and (ii) in Sec. IVB.

Another point of interest for the non-trivial stable stationary solution $c^{S}$ is its dependence on the maximum number of offsprings $N_{\text {off }}$. One can retain two important aspects, namely:

(a) At low concentrations (where the exclusion hypothesis is practically irrelevant), the survival in a highly- 
demanding environment (large $\mathcal{S}$ ) is more efficiently ensured when more descendants are born at each mating. (b) In a medium- and low-demanding environment, $c^{S}$ is increasing with decreasing $N_{\text {off }}$ (for a given value of $\mathcal{S})$. Indeed, a population that gives birth to fewer descendants at each mating has to compensate the death rate (that is fixed by $\mathcal{S}$ ) through more mating, and thus through a larger concentration. However, this concentration cannot exceed a certain value, since, on one hand, the individuals have to move in order to mate (and the corresponding jump probability $R_{1}(c)$ is decreasing as $1-c^{4}$ ), and, on the other hand, there has to be "enough room" for the born offsprings to be put-down (indeed, the corresponding probability $R_{2}(c)$ is rapidly-decaying as $\approx(1-c)^{N_{\text {off }}}$ for large values of $c$ ). The stationary value $c^{S}$ results thus from the balance between these contradictory tendencies.

\section{B. Time-periodic habitat}

Suppose that the environment is changing periodically in time, and consider, as the simplest possibility:

$$
\varphi(t)=0.5+A \sin \left(2 \pi \frac{t-t_{\mathrm{i}}}{T_{p}}\right) \Theta\left(t-t_{\mathrm{i}}\right) .
$$

Here $A$ denotes the amplitude of the environmental perturbation $(0<A \leqslant 0.5), T_{p}$ is its period, and $t_{\mathrm{i}}$ is the moment of onset of the perturbation; $\Theta$ is the Heaviside step function. The mean trait of the population is still supposed constant, $\langle z\rangle=0.5$, and the selection has a certain given value $\mathcal{S}=\mathcal{S}_{\mathrm{i}}$.

This situation is formally equivalent to that of a population of constant mean trait in a constant environment, $\langle z\rangle=\varphi=0.5$, but for which the $\mathcal{S}$ oscillates, with a period $T_{p} / 2$, as:

$$
\mathcal{S}(t)=\frac{\mathcal{S}_{\mathrm{i}}}{1-A\left|\sin \left(2 \pi \frac{t-t_{\mathrm{i}}}{T_{p}}\right)\right|} \Theta\left(t-t_{\mathrm{i}}\right) .
$$

This point of view allows us to explain easily the behavior of the concentration depending on the value of $\mathcal{S}_{\mathrm{i}}$ and on the parameters $A$ and $T_{p}$ of the perturbation.

For $\mathcal{S}_{\mathrm{i}} \geqslant \mathcal{S}_{c}$, the population dies after a time of the order of $T_{\text {rel }}^{0}$, so this case is not particularly interesting.

Let us suppose that $\mathcal{S}_{\mathrm{i}}<\mathcal{S}_{c}$, and that $c(0)$ is in the basin of attraction of the stable solution $c_{\mathrm{i}}^{S}$ that corresponds to $\mathcal{S}_{\mathrm{i}}$. After a time of the order of $T_{\text {rel }}^{S}$, the concentration relaxes to $c_{\mathrm{i}}^{S}$. We suppose that the perturbation of the selection parameter is applied after reaching this state, i.e., $t_{\mathrm{i}} \gg T_{\mathrm{rel}}^{S}$. After that, $\mathcal{S}(t)$ starts to grow, and at $t_{i}+T_{p} / 4$ it is reaching the final maximum value $\mathcal{S}_{\mathrm{f}}=\mathcal{S}_{\mathrm{i}} /(1-A)$; then it decreases during $T_{p} / 4$ till reaching the initial value $\mathcal{S}_{\mathrm{i}}$, etc. Two situations may appear:
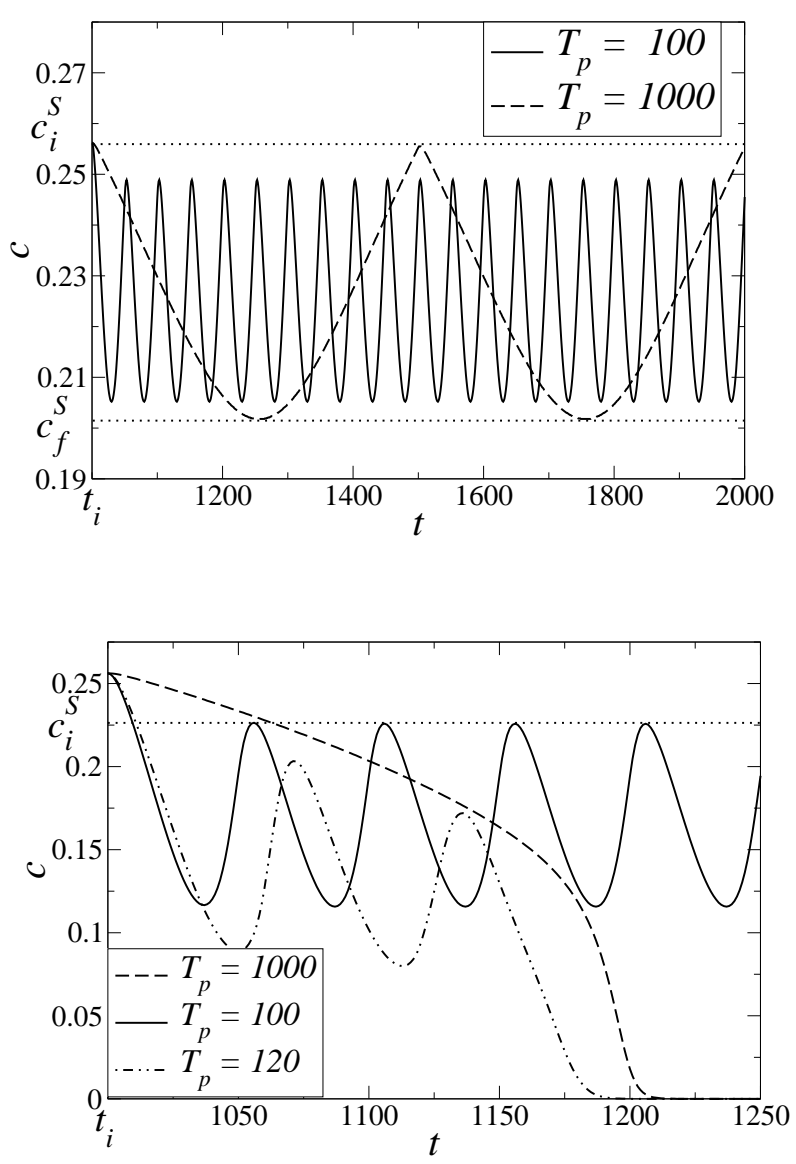

FIG. 2: MF: Possible temporal evolutions of the concentration $c(t)$ in a periodically-changing habitat for different values of $A$ and of $T_{p}$. The other parameters are $c(0)=0.7, \mathcal{S}_{\mathrm{i}}=0.3$ and $t_{\mathrm{i}}=1000$. The upper panel refers to the case (i) described in the text and is obtained for $A=0.2$. The lower panel corresponds to the case (ii) in the text, and is obtained for $A=0.35$.

Case (i): $\mathcal{S}_{\mathrm{f}}<\mathcal{S}_{c}$.

The amplitude $A$ of the perturbation is such that $\mathcal{S}(t)$ never overcomes $\mathcal{S}_{c}$. Then the nontrivial attractor $c^{S}$ (with $c^{S} \leqslant c_{\mathrm{i}}^{S}$ ) never ceases to exist, and $c$ always remains in its basin of attraction. The population never dies, and its concentration is oscillating in time, with a period $T_{p} / 2$ and an amplitude that is generally determined by both $A$ and $T_{p}$, see the upper panel of Fig. 2 ,

Case (i.a). If the oscillations are very slow, $T_{p} \gg T_{\text {rel }}^{S}$ for all the values of $\mathcal{S}_{\mathrm{i}} \leqslant \mathcal{S} \leqslant \mathcal{S}_{\mathrm{f}}$, then at each moment the concentration relaxes to the instantaneous value of $c^{S}$, see the upper panel of Fig. 3. Thus, the concentration oscillates between the two extremum possible values $c_{\mathrm{i}}^{S} \geqslant c(t) \geqslant c_{\mathrm{f}}^{S}$ (where $c_{\mathrm{i}, \mathrm{f}}^{S}$ are the equilibrium values corresponding to $\mathcal{S}_{\mathrm{i}, \mathrm{f}}$ ).

This situation is also illustrated in the second panel of Fig. 3 in terms of the potential $U_{\text {sel }}(c)$ that is driving the 
evolution of $c(t)$, see Eq. (9). When $\mathcal{S}(t)$ is oscillating between $\mathcal{S}_{\mathrm{i}}$ and $\mathcal{S}_{\mathrm{f}}$, the profile of the potential $U_{\text {sel }}(c)$ is also changing periodically. For a very slow evolution of $U_{\mathrm{sel}}(c)$, the representative point of the concentration $c(t)$ is practically always "stuck" to the minimum of the instantaneous potential.

Case (i.b) If the oscillations of the perturbation are rapid, i.e., $T_{p}$ is comparable to $T_{\text {rel }}^{S}$, then the concentration does not have time to relax to the instantaneous value of the attractor $c^{S}$, and the amplitude of its oscillation is therefore smaller than in case (a), see the upper panel of Fig. 2. This amplitude is strongly dependent on $T_{p}$, and for very small values of $T_{p}$ the concentration reaches practically a constant value. Thus, for a very rapidly-changing environment, one approaches a behavior that is reminiscent of the one appearing in the limit of a constant environment $A=0$.

The third panel of Fig. 3 represents this situation in terms of the competition between, on one hand, the relaxation of the representative point $c(t)$ to the instantaneous minimum of $U_{\text {sel }}(c)$, and on the other hand, the rapid variation of the position of this minimum: $c(t)$ lies always "behind" the minimum.

Case (ii): $\mathcal{S}_{\mathrm{f}}>\mathcal{S}_{c}$.

Suppose that the amplitude $A$ of the perturbation allows the selection to overcome the critical value, i.e., $\mathcal{S}_{\mathrm{f}} \geqslant \mathcal{S}(t)>\mathcal{S}_{c}$ during a certain interval of time $\tau$. Then the attractor $c^{S}$ ceases to exist during this interval, and the concentration tends to relax to the absorbing state $c=0$, on a time scale of the order of $T_{\text {rel }}^{0}$.

Case (ii.a) If $\tau \gg T_{\mathrm{rel}}^{0}$ (the case of a slow perturbation), then the population will die during the interval $\tau$. The corresponding evolution of the concentration is represented in the lower panel of Fig. 2. see also the upper and middle panels of Fig. 4 for an illustration.

Case (ii.b). If, however, $\tau \lesssim T_{\text {rel }}^{0}$ (rapid perturbation), then the population may survive till the end of the interval $\tau$. At this moment, the attractor $c^{S}$ starts to exist again; if not already out of its basin of attraction, the concentration tends to relax to it, till $\mathcal{S}$ reaches again the initial value $\mathcal{S}_{\mathrm{i}}$ and the cycle starts all over again. The behavior of the population in this case may be either oscillating (after a transient regime), or may present damped oscillations to the absorbing state $c=0$ (the latter regime, however, is appearing for extremely narrow intervals of the parameters). This is illustrated in the lower panel of Fig. 2 and also schematically in the upper and lower panels of Fig. 4.

Another important characteristic of the system is the extinction time (ET), which represents the time it takes for the population to get extinct, i.e., to reach the absorbing $c=0$ state. The phase transition "extinct-alive" is reflected in the behavior of the ET, which is finite for SPs below the critical value and becomes infinite at the
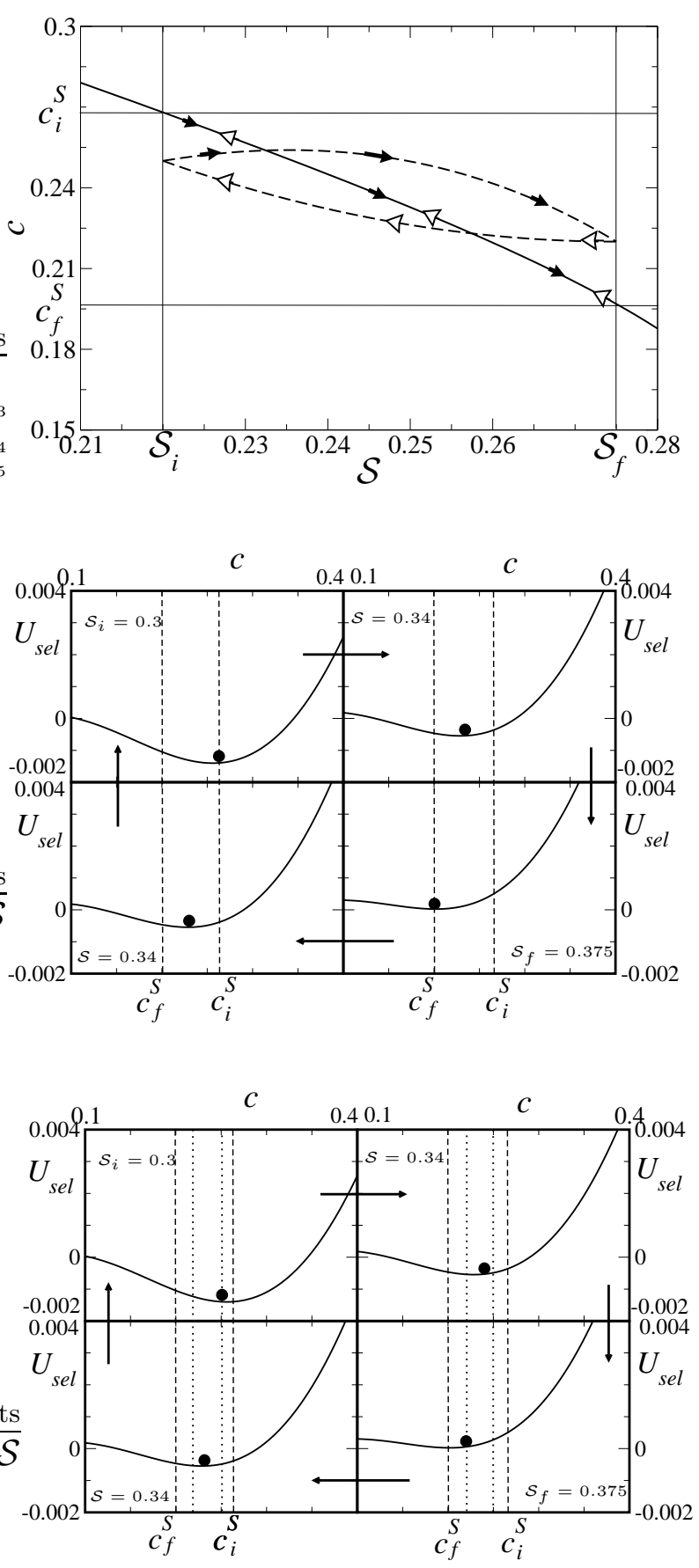

FIG. 3: MF: The upper panel describes schematically the trajectory of the system in the plane concentration-selection for the case (i) as discussed in the text, for $\mathcal{S}_{\mathrm{i}}=0.3$ and $A=0.2$ (with $\varphi=\langle z\rangle=0.5$ ). The continuous line corresponds to the case (i.a) (slow perturbation), while the dashed line represents case (i.b) (rapid perturbation). Black arrows refer to the increase of $\mathcal{S}$, and the white ones to the decrease of $\mathcal{S}$. The middle panel illustrates the behavior of the representative point $c(t)$ (the big black dot) in terms of the oscillating potential $U_{\text {sel }}(c)$ for case (i.a), and the lower panel corresponds to case (i.b). The thick arrows sketch the sense of evolution of $U_{\text {sel }}(c)$; the values of $\mathcal{S}$ corresponding to the represented profiles of $U_{\text {sel }}(c)$ are also indicated. 

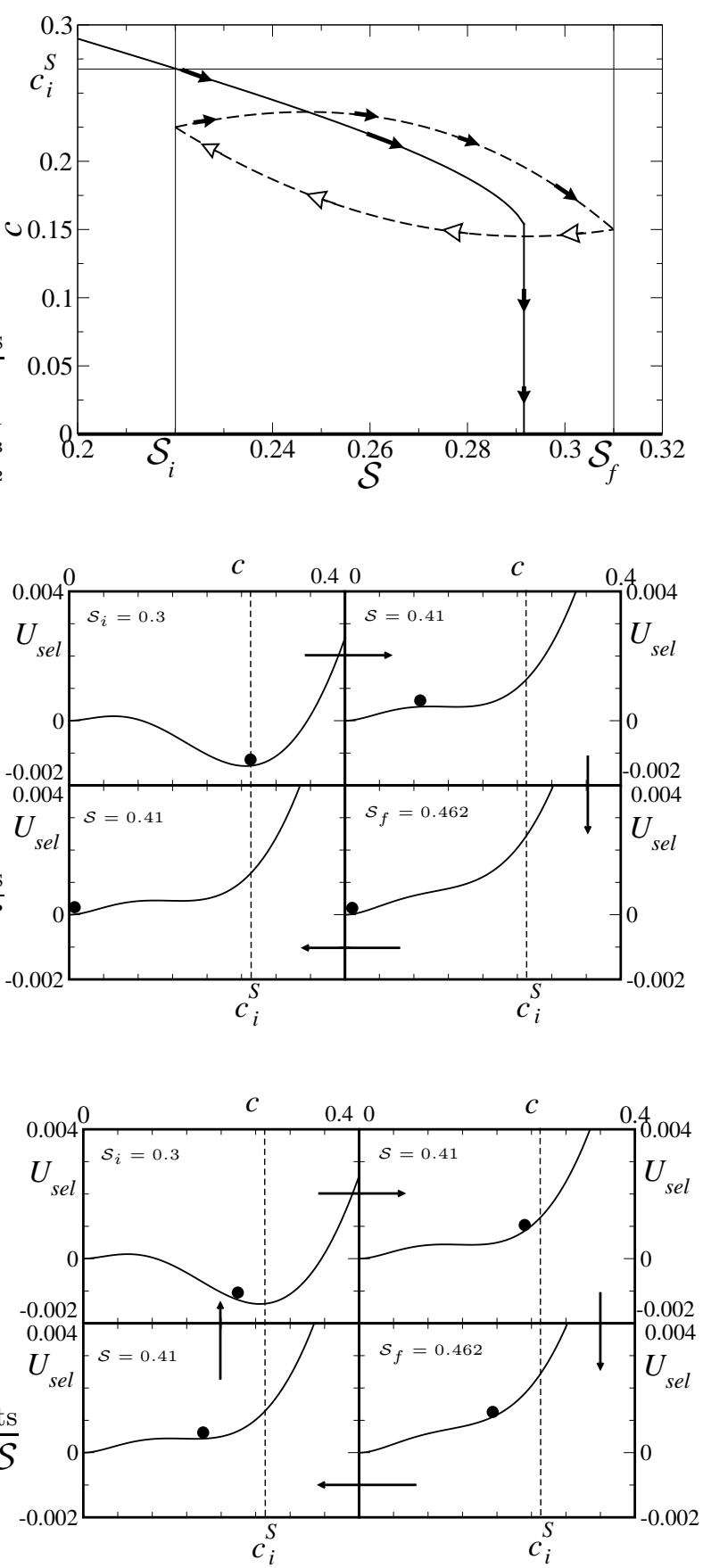

FIG. 4: MF: Upper panel: schematic representation of the trajectory of the system in the plane concentration-selection for case (ii) of the main text, for $\mathcal{S}_{\mathrm{i}}=0.3$ and $A=0.3$ (with $\varphi=\langle z\rangle=0.5$ ). The continuous line corresponds to case (ii.a) (slow perturbation), and the dashed line represents case (ii.b) (rapid perturbation). Black/white arrows correspond to the increase/decrease of $\mathcal{S}$. Middle and low panels: the behavior of the representative point $c(t)$ (big black dot) when the profile of the potential $U_{\text {sel }}(c)$ is evolving in the sense sketched by the thick black arrows. Middle panel: case (ii.a). Lower panel: case (ii.b). The values of $\mathcal{S}$ corresponding to the represented profiles of $U_{\text {sel }}(c)$ are also indicated. critical value of $\mathcal{S}_{i}$, as illustrated in Fig. 5 .

For a constant environment, the ET is finite as long as $\mathcal{S}_{\mathrm{i}}>\mathcal{S}_{c} \approx 0.417$, but it tends to infinity for $\mathcal{S}_{\mathrm{i}}<\mathcal{S}_{c}$ (provided, of course, that the initial concentration $c(0)$ is not in the basin of attraction of the absorbing state).

For the case of a time-periodic environment, the ET may also be infinite, or finite. In the first case the concentration $c(t)$ has an oscillatory behavior, while in the latter it tends to the absorbing $c=0$ state. The critical value of $\mathcal{S}_{\mathrm{i}}$ that separates these two situations depends in general on both $A$ and $T_{p}$, and it is always comprised between $\mathcal{S}_{c}(1-A)$ and $\mathcal{S}_{c}$. For very slow perturbations, the critical value of $\mathcal{S}_{i}$ approaches $\mathcal{S}_{c}(1-A)$, which is the one that corresponds to a constant environment with $\varphi=0.5+A$. Note, however, that increasing $T_{p}$ to the limit $T_{p} \rightarrow \infty$ (for a fixed $A$ ) does not imply that the general behavior of the system "converges" to the one corresponding to a constant environment with $\varphi=0.5+A$. This point is illustrated in Fig. 5, the profiles of ET corresponding to increasing $T_{p}$ values do not approach the profile for a constant environment with $\varphi=0.5+A=0.8$.

For rapid fluctuations the critical value of $\mathcal{S}_{i}$ depends strongly on $A$ and $T_{p}$. However, in the $T_{p} \rightarrow 0$ limit (infinitely-fast fluctuations), it approaches the value $\mathcal{S}_{c}$. More generally, the very fast oscillations of the optimum (at arbitrary but fixed $A$ ) are "smeared out", simply because the relaxational dynamics of the system cannot follow these optimum fluctuations. Thus the behavior of the system converges to the one corresponding to the constant environment $\varphi=0.5$.

For comparison, we also included in Fig. 5 the ET for the case when the optimum presents an abrupt jump from $\varphi=0.5$ at $t<t_{i}$ to $\varphi=0.5+A$ at $t>t_{i}$, a situation that could model a catastrophic change in the environment. The interpretation of the corresponding curve "III" is obvious. It shows, for example, that a population that could survive a rather rapid but smooth variation of the optimum cannot survive a catastrophe.

This detailed discussion clearly illustrates the delicate interplay between the time-scale and amplitude of the perturbation, on one hand, and the intrinsic time-scales and nonlinearities of the unperturbed dynamics, on the other hand. This interplay is determinant even in this simplified MF-type of approach; we are thus expecting it to play a prominent role for the case of a more microscopic, individual-based modeling, for which the fluctuations (in particular, the mutations) are enriching the dynamics even further.

\section{IBM-TYPE SIMULATIONS}

Let us turn now to the results obtained through numerical simulations in the IBM-type of approach described in Sec. IIIB One is apriori entitled to expect a richer dynamics in this microscopic approach, since: (a) on one hand, the mutation is definitely playing a role, and $\mathcal{M}$ represents the second control parameter besides the se- 


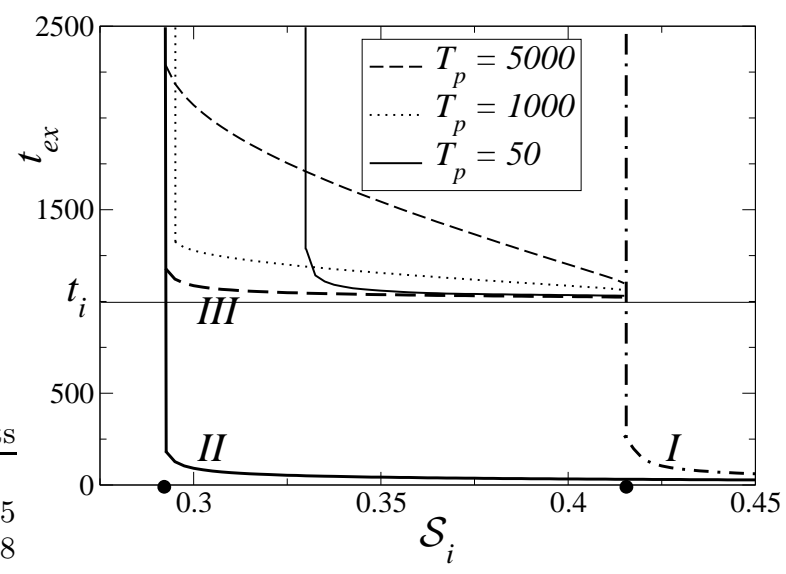

FIG. 5: MF: Extinction time $t_{\mathrm{ex}}$ as a function of the initial selection parameter $\mathcal{S}_{\mathrm{i}}$ for different periods $T_{p}$ of the perturbation. The amplitude of the perturbation is fixed to $A=0.3$. The critical values of $\mathcal{S}_{\mathrm{i}}$ are comprised between the extremal points $\mathcal{S}_{c}(1-A) \approx 0.292$ and $\mathcal{S}_{c} \approx 0.417$ (represented by the big dots on the abcise). The continuous thick curve labelled $I$ gives the ET as a function of $\mathcal{S}_{i}$ for the case of a constant environment with $\varphi=0.5$, while the dashed-dotted curve labelled $I I$ is obtained for a constant environment with $\varphi=0.8$. Finally, the thick dashed curve labelled $I I I$ corresponds to an abrupt jump in the optimum, from $\varphi=0.5$ for $t<t_{i}$ to $\varphi=0.5+A=0.8$ at $t>t_{i}$. The other parameters are $\langle z\rangle=0.5, t_{\mathrm{i}}=1000$, and $c(0)=0.7$.

lection $\mathcal{S}$; (b) on the other hand, the fluctuations and the finite size of the system can influence in a nontrivial way the global dynamics of this highly-nonlinear system.

The initial conditions for the system, as already mentioned in Sec. IIIB, are represented by the positions of the individuals on the nodes of the lattice, as well as by their individual traits. If not explicitly stated otherwise, we shall consider hereafter that initially the $N(0)$ individuals are randomly, uniformly distributed on the lattice, with a given initial concentration $c(0)=N(0) / L^{2}$; also, their traits are randomly, uniformly distributed in the interval $[0,1]$. The case when the $N(0)$ individuals have the same trait was also studied. The initial concentration is fixed throughout to the rather larg value $c(0)=0.7$. The role of $c(0)$ on the survival of the population, and the existence of a critical initial concentration (the so-called minimum viable population concentration) below which the population gets extinct for given environmental conditions are well-known issues, see e.g. [29, 30, 31], and we shall not address them further here. In the foregoing we shall keep $N_{\text {off }}=6$.

The control parameters that determine the dynamics of the system are the $\mathrm{SP}-\mathcal{S}$ and the $\mathrm{MA}-\mathcal{M}$. In the case of a variable environment, as described by Eq. (10), one has to consider two further control parameters, namely the amplitude $A$ and period $T_{p}$ of the perturbation of the optimum. The onset time of the optimum's variation is fixed to $t_{\mathrm{i}}=1000 \mathrm{MCS}$, and it is sufficiently large such that the system relaxes to a stationary state in the interval between $t=0$ and $t=t_{i}$.

Even when the above parameters, as well as the initial concentration and mean trait are fixed, there are still various possible realizations of the system's evolution, that are induced by the different sources of stochasticity which are present in the system, namely, (a) the randomness in the initial conditions (random positions of the individuals on the lattice, and/or to their randomly-distributed individual traits); (b) the stochastic aspect in each individual's death; (c) the random, diffusion-like jumps of the individuals on the lattice; (d) the mating and the stochasticity elements in the progeny-birth, including the mutation in the trait of the resulting offsprings.

Some measurable quantities [(i) to (iv) below] that can characterize in an efficient way the behavior of the populations can be cast into two categories: those referring to a single realization of the population's evolution, and those related to an ensemble of such realizations.

For a single realization, these quantities are, respectively:

(i) The "global" concentration $c(t)=N(t) / L^{2}$, where $N(t)$ is the number of individuals at time $t$.

(ii) The distribution of the individual traits $P(z, t)$ and, in particular, the time-dependent value of the mean trait,

$$
\langle z(t)\rangle=\int_{0}^{1} d z z P(z, t)=\frac{1}{N(t)} \sum_{i=1}^{N(t)} z_{i} .
$$

Another related quantity, currently used by biologists [20], is the mean maladaptation,

$$
\langle\mu(t)\rangle=\int_{0}^{1} d z|z-\varphi(t)| P(z, t)=\frac{1}{N(t)} \sum_{i=1}^{N(t)}\left|z_{i}-\varphi(t)\right|,
$$

which represents a measure of the deviation of the population from the instantaneous optimum imposed by the habitat.

(iii) The distribution of the individual extinction probabilities $\Pi(p, t)$, that is directly related to $P(z, t)$. Indeed, note that $\Pi(p, t)|d p|=P(z, t)|d z|$, from which one finds, using Eqs. (11) and (2) that:

$$
\Pi(p, t)=\frac{\mathcal{S}}{(1-p) \ln ^{2}(1-p)} P(z, t) .
$$

For an ensemble of realizations, the main element of interest is represented by:

(iv) The mean extinction time (MET) $\overline{t_{\mathrm{ex}}}$ for a fixed set of parameters. Indeed, due to the intrinsic randomness of a population's evolution, the extinction time is a stochastic variable, that differs from one realization of the dynamics to another. As such, one can consider the associated probability distribution funtion of the extinction time, $\mathcal{T}\left(t_{\text {ex }}\right)$ and, in particular, its first moment,

$$
\overline{t_{\mathrm{ex}}}=\int_{0}^{\infty} d t_{\mathrm{ex}} t_{\mathrm{ex}} \mathcal{T}\left(t_{\mathrm{ex}}\right) .
$$




\section{A. Constant environment}

Let us consider first a situation that is analogous to the one described in Sec. IVA, namely a constant environment with $\varphi=0.5$, in which evolves a finite population with random initial conditions, which means random positions of the individuals on the nodes of the lattice, as well as randomly distributed individual traits with $\langle z(t=0)\rangle=0.5$.

The main question is whether the phase transition "extinct-alive" encountered in the MF approach is destroyed by the fluctuations, or is it still appearing in this IBM description. The situation is of course more complicated, due to the effects of the mutation, i.e., due to the existence of the second control parameter represented by the amplitude $\mathcal{M}$ of the mutation (see Sec. IIIB). A related question is, naturally, how is the mutation affecting the appearance and location of a phase transition.

\section{Evolution of the concentration $c(t)$}

An indication on a possible phase transition is obviously offered by the asymptotic behavior of the concentration $c(t)$ as a function of the selection parameter $\mathcal{S}$, for given initial conditions and a fixed value of $\mathcal{M}$. Indeed, (a) for "large-enough" selection pressures, $c(t)$ is decreasing, with random fluctuations, till the absorbing $c=0$ state; (b) for "small-enough" values of $\mathcal{S}$, in the long-time limit the concentration $c(t)$ reaches a regime of stationary fluctuations around a non-zero constant mean value $c^{S}$. These results clearly point to the existence of a phase transition "extinct-alive". Of course, one has to be aware that a "true" phase transition can only appear in the thermodynamic limit of an infinite population on an infinite-size lattice, see also [27]. In particular, due to the finiteness of the system, the transition is not abrupt (i.e., there is not a clear-cut critical value $\mathcal{S}_{c}$ ), and the frontier between the basins of attraction of the absorbing state $c=0$ and of the non-trivial state $c^{S}$ is also spread out.

\section{Behavior of the MET $\overline{t_{\mathrm{ex}}}$}

Another good indication about the existence of the phase transition "extinct-alive" is offered by the behavior of the MET $\overline{t_{\mathrm{ex}}}$ as a function of $\mathcal{S}$. The main results are resumed in Fig. 6, that indicates a sudden, very abrupt increase in $\overline{t_{\mathrm{ex}}}$ with decreasing $\mathcal{S}$, which is the signature of the phase transition. Moreover, Fig. [6 is illustrating the way the mutation $\mathcal{M}$ modifies the location of the transition.

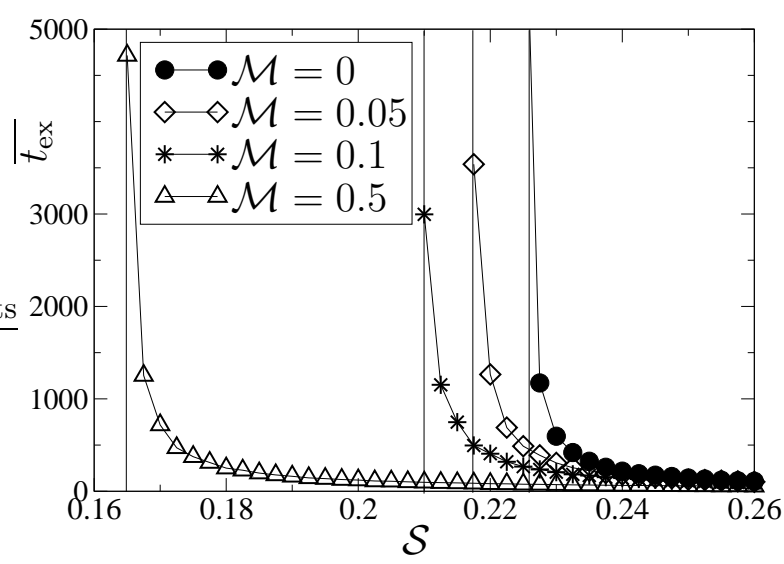

FIG. 6: IBM approach: The mean extinction time $\overline{t_{\mathrm{ex}}}$ in a constant environment as a function of the selection parameter $\mathcal{S}$, for different values of the mutation $\mathcal{M}$. The vertical lines indicate the estimated locations of the critical selections for each value of $\mathcal{M}$. The other parameters are $c(0)=0.7, L=$ $100, \varphi=0.5$, and the average was taken over 100 realizations.

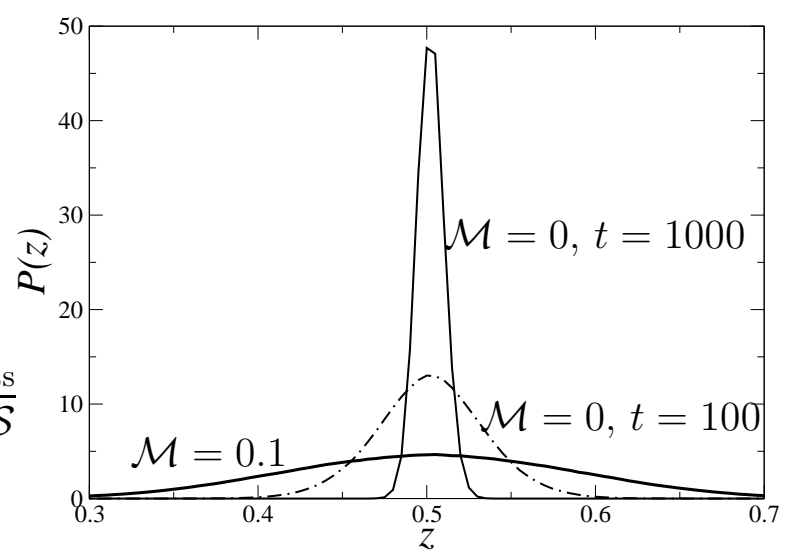

FIG. 7: IBM approach: Snapshots of the probability distribution function of the trait $P(z, t)$ at two times $t=100$ and $t=1000$. The thin continuous and dashed-dotted lines refer to the population without mutation, $\mathcal{M}=0$. The thick line corresponds to the population with $\mathcal{M}=0.1$, for which $P(z, t)$ reaches after some $10 \mathrm{MCS}$ a stationary profile. The values of the other parameters are $\mathcal{S}=0.13, c(0)=0.7, L=4000$ (for a good statistics), and $\varphi=0.5$.

\section{Role of the mutation}

It appears from Fig. 6 that increasing mutation amplitude $\mathcal{M}$ is unfavorable to the survival of a population in a constant environment and the transition "extinct-alive" is shifted to lower values of $\mathcal{S}$. The argument is that a larger $\mathcal{M}$ implies a larger chance for an offspring to be ill-adapted to the constant environment, even though the parents might be well-adapted. 
To get a more quantitative picture of the various factors that are involved in this result let us consider a given population and focus on the behavior of the probability distribution function of the trait $P(z, t)$ [38]. Let us fix the value of the selection - for example, $\mathcal{S}=0.165$, such that, according to Fig. 6, a population without mutation $(\mathcal{M}=0)$ will survive (i.e., its asymptotic regime will correspond to a constant mean concentration $c^{S} \neq 0$ ). Let us compare it with the case of a population with non-zero mutation $\mathcal{M}=0.1$, i.e., a population that, again according to Fig. 6] will not survive the SP. Then, according to Fig. 7] for the population without mutation, $\mathcal{M}=0$, the distribution of the trait $P(z, t)$ is narrowing towards a $\delta$-peak around $z=\varphi=0.5$ by a progressive elimination, due to the SP, of the ill-adapted individuals. Moreover, the offsprings of well-fitted parents will be also well-fitted, since their trait is a pure inheritance equal to the mean trait of the parents. On the contrary, for the case of the population with $\mathcal{M}=0.1$ one notices that, after a rather rapid transient (a few 10s MCS), the distribution of the traits remains practically constant. So, although the illadapted individuals are eliminated in priority by the SP (the distribution of the traits tends to be narrowed by the selection), at each mating the traits of the offsprings are randomized again by the mutation (the distribution of the traits tends to be broadened by the mutation). The resulting stationary distribution, that has a finite width of the order of $\mathcal{M}$, is a balance between these two tendencies.

Therefore the population with $\mathcal{M}=0.1$ is at each moment more vulnerable than the population with $\mathcal{M}=0$, and may thus die even if the population without mutation is surviving.

One notices that the evolution of the population, even in the case $\mathcal{M}=0$, is much more complex than that described by the MF approach. In particular, the dynamics of the concentration and of the distribution of the individual traits are strongly inter-related. As long as $\mathcal{M}=0$, they are taking place on a comparable time scale, but it is difficult to assimilate this time scale with the one of the simple relaxational processes that appear in the MF description. However, when $\mathcal{M} \neq 0$, the evolution of the concentration and that of $P(z, t)$ take place, in general, on different time scales. For example, for the situation of a population with $\mathcal{M}=0.1$ considered in Fig. 7, the relaxation of $P(z, t)$ to its asymptotic profile takes place during a short transient time of the order of $10^{2} \mathrm{MCS}$, while the relaxation of the concentration $c(t)$ to the absorbing state $c=0$ is very slow, with a characteristic time of the order $10^{5} \mathrm{MCS}$, that can be estimated from the asymptotic part of the plot $c(t)$.

As can be seen, the mutation is introducing essentially new features in the dynamics, even in the simplest context of a constant environment. In particular, the characteristics of the population (like concentration, trait and individual extinction probabilities) can evolve on different time scales, that are sensitive to both control parameters of selection $\mathcal{S}$ and mutation $\mathcal{M}$. This fundamental role of the mutation is not at all accounted for in the MF description.

After discussing these essential qualitative differences that appear between the MF and the IBM results, let us turn to the problem of the calibration of the parameters corresponding to the two levels of modeling. One has to retain that our MF description is not the result of a rigorous coarse-graining procedure applied to the IBM-model. As such, besides the problem of the correspondence of the unit-time already mentioned in Sec. II. the control parameter of selection $\mathcal{S}$ that appears in both approaches may present some calibration problems, too. Moreover the difficulty in establishing a quantitative correspondence between the two aproaches is actually far from being a simple rescaling problem.It is related to the role of the spatial correlations between the individuals (like, for example, possible clustering effects), role that is completely neglected in the MF approach. Let us consider just two aspects in order to illustrate this point: (a) It seems that these correlations tend to increase the value of the asymptotic concentration $c^{S}$, however they are contributing to reduce the survival chances at increasing SP as compared to the MF. (b) The comparison between the role that the maximum number of offsprings $N_{\text {off }}$ is playing in the MF description and in the IBM one indicates that in both cases the critical value of the selection $\mathcal{S}_{c}$ is increasing with $N_{\text {off }}$. However, the concentration $c^{S}$ of the stationary non-absorbing state is decreasing with $N_{\text {off }}$ in the MF description, while it is increasing with $N_{\text {off }}$ in the IBM approach.

\section{B. Time-periodic changes of the environment}

Let us now turn to the case of a time-dependent habitat, whose optimum varies in time according to Eq. (10).

\section{Phase diagram: role of selection $\mathcal{S}$ and mutation $\mathcal{M}$}

Consider the location of the phase transition point "extinct-alive", i.e., the critical value of the SP for a population with given initial conditions. This critical point depends not only on the amplitude $A$ and period $T_{p}$ of the optimum variation, but also on $\mathcal{M}$ - an aspect which of course cannot be captured by the MF description. One is thus led to consider the phase diagram in the plane of the characteristic parameters $\mathcal{S}$ and $\mathcal{M}$, see Fig. 8 ,

Several characteristics are emerging from the analysis of these critical curves, namely:

(a) The phase diagram is very little affected by the actual value of the optimum $\varphi$ of a constant environment. Indeed, as illustrated in Fig. 8, the curves for $\varphi=0.5$ and $\varphi=0.8$ present little differences.

This result holds provided that the population has initially randomly-distributed individual traits. In this case, whatever the constant value of the optimum $\varphi$, 


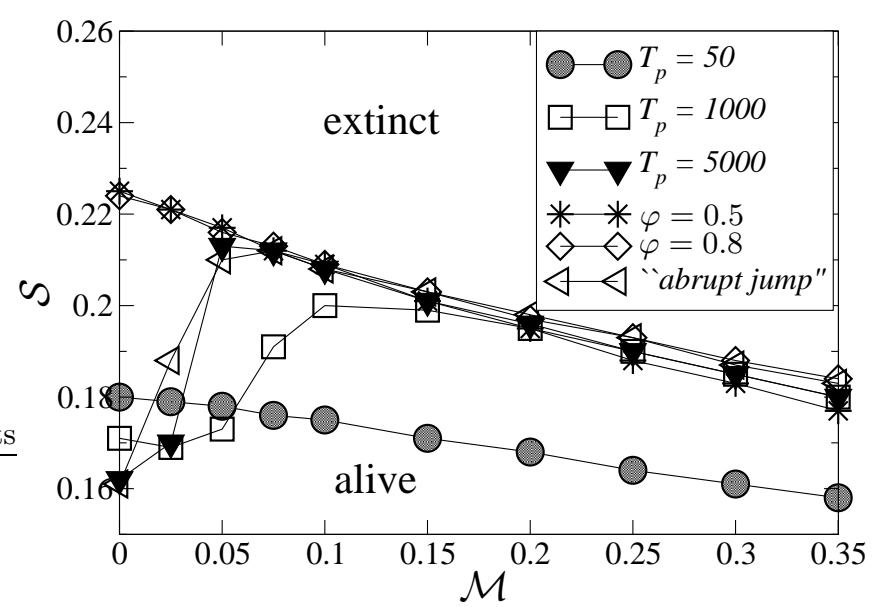

FIG. 8: IBM approach: The phase diagram "extinct-alive" in the $\mathcal{S}$ and $\mathcal{M}$ plane, for various periods $T_{p}$ of the optimum oscillation. For comparison we also presented the results for a constant environment with $\varphi=0.5$, respectively $\varphi=0.8$, as well as for an abrupt jump of the optimum (at $t_{i}=1000$ ) between these two values. The average was done over 100 runs and the vertical size of the symbols is of the order of the estimated error bars. The continuous lines are just a guide for the eye. The values of the other parameters are $A=0.3$, $c(0)=0.7$, and $L=100$.

a fraction of the initial population is (more or less) illadapted and will be progressively eliminated. Also, the descendancy will become progressively more and more fitted to the environment. As such, in the long run the distribution of the trait of the population will become peaked around $\varphi$, with a variance of the order of $\mathcal{M}$, and the corresponding distribution of the individual extinction probabilities will be essentially the same for all constant values of $\varphi$. The small differences that appear, especially for small values of $\mathcal{M}$, in the critical curves for $\varphi=0.5$ and $\varphi=0.5+A$ are due to the way we prescribed the trait of a progeny, according to Eq. (3). When starting from randomly-distributed initial individual traits, the heredity part tends to "push" the descendancy towards a mean trait equal to 0.5 ; thus the relaxation towards the asymptotic profile of the distribution of the individual extinction probabilities will be slower for $\varphi=0.5+A$ than for $\varphi=0.5$. This makes the population with $\varphi=0.5+A$ a little bit more sensitive to the SP.

Also, as already mentioned in the previous section, for a constant environment any modification of the status quo of the population due to the mutation is "bad", and the critical value of $\mathcal{S}$ decreases monotonously with $\mathcal{M}$.

(b) Consider now a population with initially equal individual traits $z_{i}=0.5$ and put it in a constant environment with $\varphi=0.5+A$. For small values of the mutation $\mathcal{M}$ the randomness in the descendancy traits cannot overcome the misfit inherited from the parents. It is thus clear that the population will get extinct for smaller values of $\mathcal{S}$ than in the cases discussed above. This point is also reflected in the behavior of the MET. The result for $\mathcal{M}=0$ is in agreement with the MF case, see Fig. 5 .

However, for large values of $\mathcal{M}$ the inherited traits of the offspring are greatly changed by mutations and the progeny adapts to the constant environment with $\varphi=$ $0.5+A$. The critical curve is approaching the ones for the constant optimum discussed at point (a) above.

For intermediate values of $\mathcal{M}$, inheritance and mutation are both important in the offspring traits, and the critical curve still lies below the ones corresponding to point (a). This is in agreement with the behavior of the mean extinction time, as illustrated in Fig. 10 for $\mathcal{M}=0.1$.

As such, the population is sensitive to the initial distribution of the individual traits only for small values of $\mathcal{M}$, and the memory of the initial state is lost when mutation is strong.

(c) Any variation in the optimum is harmful for the system, as indicated by the fact that all the critical curves corresponding to a variable optimum lie below the one for the constant optimum $\varphi=0.5$. This is again in qualitative agreement with the MF results.

(d1) Consider the case of a population without mutation, $\mathcal{M}=0$. The less favorable situation for such a population seems to be that of an abrupt jump in the optimum from $\varphi=0.5$ to $\varphi=0.5+A$, as compared to all the cases of smooth oscillation of the optimum around $\varphi=0.5$. This result is in qualitative agreement with the one in Ref. [28], and can be understood as follows: at $t=t_{i}$, when the perturbation of the optimum sets in, for a population with $\mathcal{M}=0$ the overhelming majority of the individuals (and thus their children) have an individual trait $z_{i}=\varphi=0.5$. An abrupt change in the optimum $\varphi$ will lead to an abrupt increase in the extinction probability of the individuals (per MCS) till its maximum possible value $p=1-\exp [-\mathcal{S} /(1-A)]$, corresponding to the given amplitude $A$. This leads to a decrease in the critical value of the selection $\mathcal{S}$. One can therefore conclude that after a sharp change in the living conditions, such as a large-scale catastrophe, even a moderate SP could be lethal for a population lacking the variety induced by mutation.

Moreover, a long period of the optimum variation is less favorable to the population survival than a shorter one. Indeed, one can argue that in the case of a large period the individuals are spending a longer time (in MCS) in an unfavorable environment, and thus have more chances to die. Recall that the absence of mutation leads to the fact that the descendancy is as ill-adapted to the environment as the parents are.

These elements are also illustrated in Fig. 9 for the dependence of the mean-extinction time on $\mathcal{S}$. 
(d2) Consider now a population with an intermediate value of the $M A$, say $\mathcal{M} \approx 0.1$. One notices that, contrary to the situation (d1) of a population with $\mathcal{M}=0$, an increase in the period $T_{p}$ of the optimum change is beneficial for the population and the critical SP is deplaced towards larger values.

Indeed, for the case of an intermediate $\mathcal{M}$, the heredity and the mutation are equally important in establishing the trait of the population. As such, if the optimum is varying slowly, the population has the time to adapt to the instantaneous value of the optimum, and "good traits" are transmitted, through heredity, to the descendancy; moreover, the randomness element due to the mutation is somehow compensating for the small variation of the optimum from one generation to the other. On the contrary, if the optimum is varying rapidly from one generation to the next one, even if the parents were welladapted at some instantaneous value of $\varphi$, they will become soon ill-adapted and transmit this misfit to their progeny through heredity. The mutation element in the offspring traits cannot compensate for the too large inherited misfit between the individual trait and the instantaneous optimum.

Therefore, a population with an intermediate mutation amplitude is more vulnerable to rapid variations in the optimum. This result is also illustrated in Fig. 10 through the behavior of the mean extinction time of a population, as a function of $\mathcal{S}$, for different values of the optimum period $T_{p}$.

(d3) Very large $M A$ is always harmful for the population's survival, whatever the period of change $T_{p}$ of the optimum. Indeed, a large MA changes so much the traits that the inheritance of good traits tends to be lost. Each generation is therefore composed of too many individuals with unfitted traits (since the "cleaning action" that the selection operated on the previous generations does not lead to a better-fitted offspring). The critical value of $\mathcal{S}$ is decreasing monotonously with $\mathcal{M}$ in the whole region of high MA.

(e) The small- $T_{p}$ curves are, in general, monotonously decreasing with increasing $\mathcal{M}$, suggesting that larger mutations, combined with rapid changes of the environment, are harmful for the population, i.e., reduce its resistance to the SP.

(f) Finally, the most spectacular element of this phase diagram is the existence of an optimal value of the mutation for intermediate values of $T_{p}$ (at fixed $A$ ), i.e., a maximum in the critical curve $\mathcal{S}-\mathcal{M}$. For this optimal mutation, the critical value of the selection is maximum, indicating that the population resists to higher SPs than for other values of $\mathcal{M}$. The value of this optimal mutation depends on the period $T_{p}$ of the optimum variation.

A crude explanation of this effect would follow these lines: Consider first a population with a small MA, that brings diversity to the phenotypic pool of the popula- tion. Through the selection, unfitted individuals tend to be eliminated, and the fitted ones survive. If the optimum varies slowly (large $T_{p}$ ), then the "good" traits, that will be passed to the next generations, plus a small mutation, remain good for some time. A small amount of mutation is therefore beneficial. If, however, $T_{p}$ is too short, then quite soon the previously good traits (that a too small mutation cannot correct, in order to accomodate them to the evolving value of the optimum !) turn out to be bad. A larger effect of the mutation is needed to ensure an optimal adaptation of the descendancy to the new values of the optimum; however, see (d3), the MA should not be "too large" either. Such effects of a beneficial range of mutation have already been observed in biological systems, see e.g. 32.

This discussion also indicates that an optimal mutation can only be found for intermediate values of $T_{p}$; too large or too small rates of changes of the optimum do not allow for the above-described effects, as illustrated in Fig. 8. The MET can be thus increased by the optimal amplitude of the mutation, for a given period $T_{p}$ of the optimum variation. This result is illustrated in Fig. 9 for the case of $T_{p}=1000$, for which the phase diagram of Fig. 8 indicates an optimal value of the mutation $\mathcal{M} \approx 0.1$. Indeed, for values of $\mathcal{M}$ above and below this opimal value, the MET (for each value of $\mathcal{S}$ ) is clearly lower than the one corresponding to the optimal mutation.

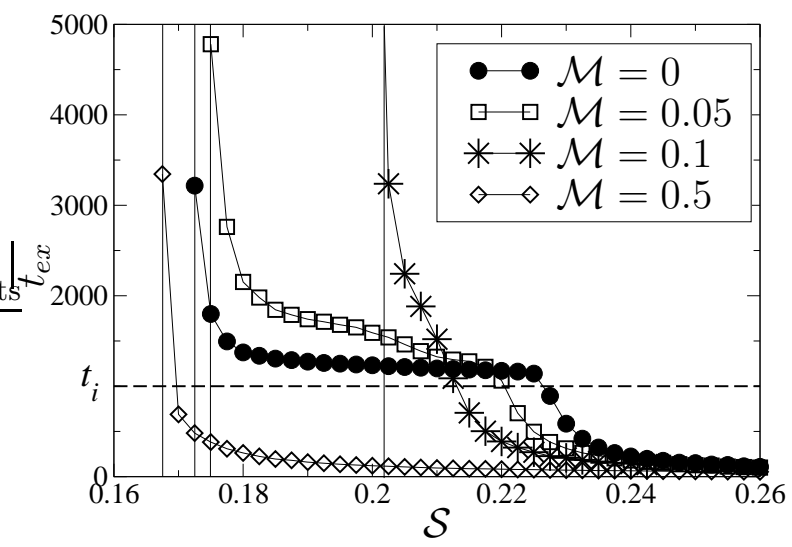

FIG. 9: IBM approach: The mean extintion time $\overline{t_{e x}}$ as a function of t $\mathcal{S}$ for different values of $\mathcal{M}$. The thin vertical lines indicate the estimated location of the transition point. The average was done over 100 populations with $c(0)=0.7$, $L=100, A=0.3, T_{p}=1000$ and $t_{\mathrm{i}}=1000$. The continuous lines are just a guide for the eye.

The signature of the phase transition "extinct-alive" can be also found in the behavior of the profile of the probability distribution function of the extinction time $\mathcal{T}\left(t_{\text {ex }}\right)$ for an ensemble of populations with fixed initial conditions $c(0),\langle z\rangle$ and given parameters $\mathcal{M}, A$, and $T_{p}$. Indeed, below the critical value of $\mathcal{S}$ the distribution of 
the extinction time is narrow-peaked around $\overline{t_{\mathrm{ex}}}$, while above the transition its profile changes, by developing a fat tail towards large values of $t_{\mathrm{ex}}$. Therefore, above the critical point $\overline{t_{\mathrm{ex}}}$ is no longer corresponding to the most representative value of $t_{\mathrm{ex}}$.

\section{Role of the period $T_{p}$ and amplitude $A$ of optimum oscillations}

The influence of the period $T_{p}$ (for a fixed value of $A$ ) on the critical value of $\mathcal{S}$ depends strongly on the value of $\mathcal{M}$. As shown in the previous paragraph, for very small or zero values of $\mathcal{M}$, a decrease in $T_{p}$ (i.e., slow perturbations of the optimum) leads to an increase in the critical value of $\mathcal{S}$.

However, for intermediate values of $\mathcal{M}$, the role of $T_{p}$ gets reversed, namely increasing $T_{p}$ (with fixed $A$ ) leads to an increase in the critical $\mathcal{S}$. This point was discussed in the previous paragraph, and it is shown in Fig. 10] of $\overline{t_{e x}}$ versus $\mathcal{S}$ for $\mathcal{M}=0.1$.

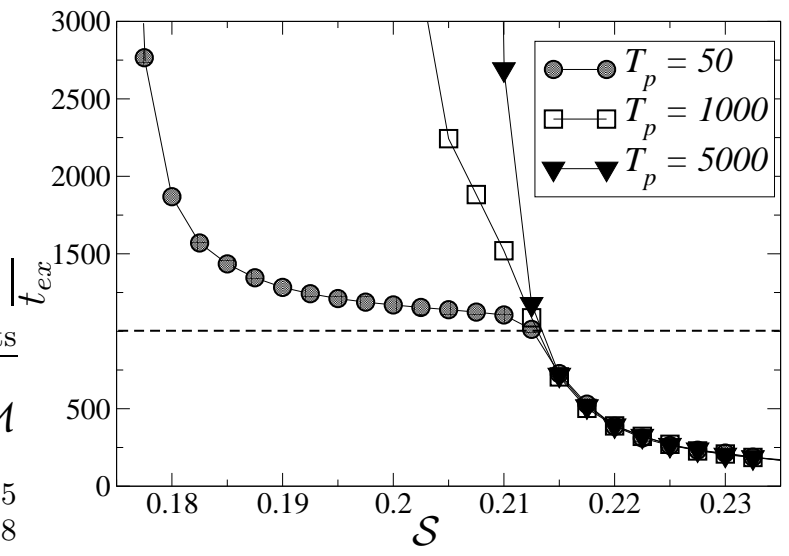

FIG. 10: IBM approach: The mean extintion time $\overline{t_{e x}}$ as a function of the selection pressure $\mathcal{S}$ for $\mathcal{M}=0.1$ (intermediate MA) and different values of $T_{p}$. The results are averages done over 100 populations with $c(0)=0.7, L=100, A=0.3$, and $t_{\mathrm{i}}=1000$.

Finally, for the case of a population with a large MA, the MET is less sensitive to the period $T_{p}$ of the optimum oscillation, as well as to the initial distribution of the individual traits than in the case of intermediate or small values of $\mathcal{M}$.

The role of the oscillation amplitude $A$ is easy to resume: increasing $A$ has a destabilizing effect on the system, i.e., it deplaces the critical curve towards smaller values of the SP, see Fig. 11, and it reduces the extinction time of the population (all the other parameters, as well as the initial conditions being kept the same). This effect, however, is more or less pronounced depending on the values of the other parameters $T_{p}$ and $\mathcal{M}$. In partic- ular, for large MAs $\mathcal{M}$ the transition point depends only weakly on the value of $A$.

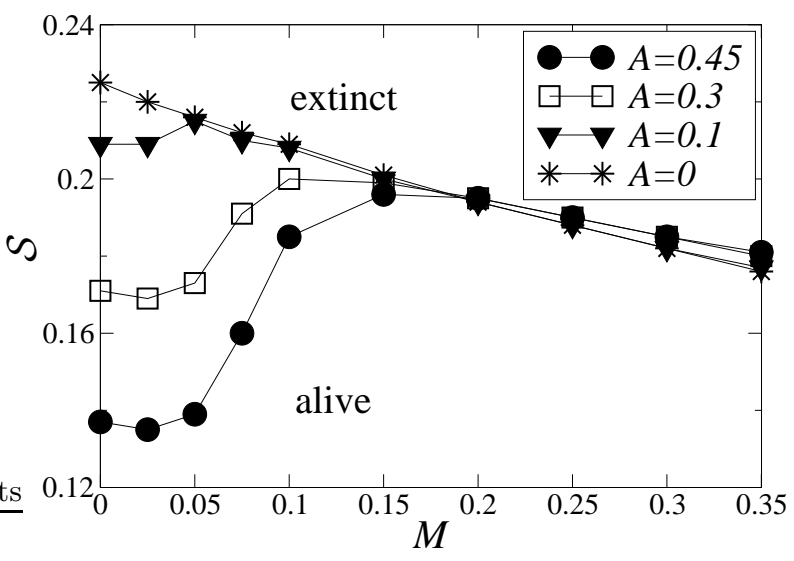

FIG. 11: IBM approach: Role of the amplitude $A$ of the optimum oscillation on the phase diagram in the $(\mathcal{S}, \mathcal{M})$ plane. The average was done over 100 populations with $c(0)=0.7$, $L=100, t_{\mathrm{i}}=1000$, and the period of optimum oscillation is $T_{p}=1000$. The continuous lines are just a guide for the eye.

\section{Influence of the system size}

The system size is playing a role on the location of the transition point, as well as on its "sharpness", see Ref. [27]. As expected on general backgrounds, the width of the transition zone is increasing with decreasing system size. Moreover, small-size populations are, on average, more vulnerable than larger-size ones, i.e., it is more probable that small-size systems get extinct for smaller values of the SP than the analogous large ones. This effect is essentially related to the larger amplitude of the relative fluctuations in the number of individuals in smaller-size systems as compared to larger-size ones; indeed, for a given mean concentration, this amplitude goes roughly as $1 / L$. This effect is well-known in biology as the demographic stochasticity effect in population extinction, see Refs. [25, 29] for further comments.

\section{Evolution of the concentration $c(t)$}

For "large" (i.e., above the transition point) values of the SP the concentration $c(t)$ tends to zero, but below the transition point the concentration reaches, after a transient regime, an oscillatory behavior of period $T_{p} / 2$, as illustrated in Fig. 12. Of course, due to the inherent stochastic nature of the dynamics, these oscillations are noisy. As in the MF case, the amplitude of these oscillations (for given $\mathcal{S}$ and $\mathcal{M}$ ) depends on both $A$ and $T_{p}$. The oscillations of the concentration are not 
in phase with the absolute value of the optimum, but exhibit a mean lag that depends on the control parameters $A, T_{p}, \mathcal{S}$, and $\mathcal{M}$ of the system. In Fig. 12 we also represented the temporal evolution of the mean population trait $\langle z(t)\rangle$, see Eq. (12). Its oscillations, of period $T_{p}$, appear only for $\mathcal{M} \neq 0$, while for $\mathcal{M}=0$ the mean trait settles down to a constant value that is equal the mean value of the optimum $(\langle z\rangle=0.5)$. Finally, Fig. 12 presents the oscillations (of period $T_{p} / 2$ ) of the mean maladaptation $\langle\mu(t)\rangle$ (Eq. (13) ), and one notices that an instantaneous minimum in the concentration corresponds to a maximum of the maladaptation. Again, the characteristics of these curves (amplitude, mean over one period, lag as compared to the optimum variation, etc.) depend on $\mathcal{S}$ and $\mathcal{M}$.

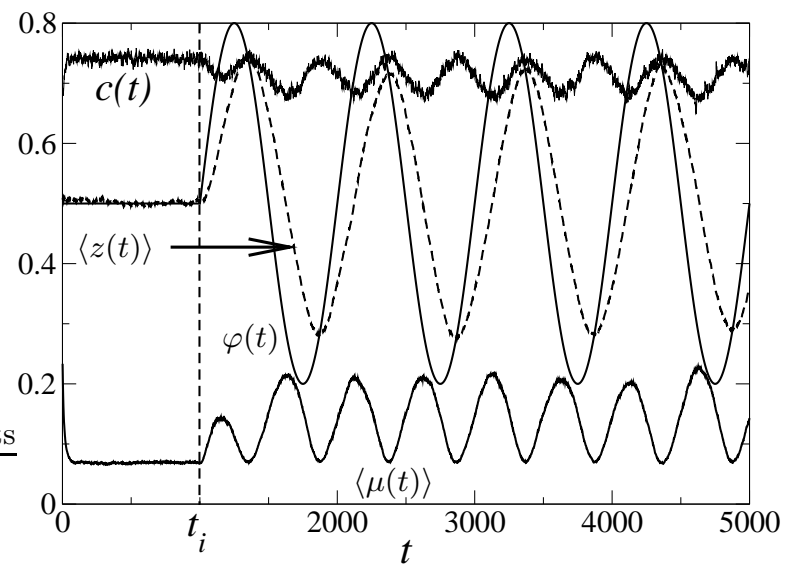

FIG. 12: IBM approach: The oscillations of the concentration $c(t)$, mean trait $\langle z(t)\rangle$ (dashed line), and mean maladaptation $\langle\mu(t)\rangle$ for a population that survives in an oscillating habitat. The values of the other parameters are $\mathcal{M}=0.1, \mathcal{S}=0.13$, $A=0.3, T_{p}=1000, t_{\mathrm{i}}=1000, L=100$, and $c(0)=0.7$. For comparison we also represented the oscillating optimum $\varphi(t)$.

\section{CONCLUSIONS AND PERSPECTIVES}

In this paper we have considered a model of singlespecies population dynamics on a $2 D$ lattice, and we analyzed the role of the selection pressure and mutation on its behavior in a periodically-changing environment. Two levels of description were considered in detail - a mean-field one, and an individual-based approach with built-in stochasticity.

The MF approach allowed us to highlight, in a rather intuitive manner, the delicate interplay between the different time-scales of the processes involved in the dynamics of this highly-nonlinear system. In particular, the influence of the amplitude and periodicity of the optimum variation on the critical value of the SP (above which the population gets extinct) was discussed in detail. However, this type of MF approach is unsuitable for describing another essential element of the population dynamics, namely the stochasticity introduced by the mutation in the descendancy traits [39].

In order to take into account the effects of the mutation, one has to appeal to a more refined level of description like the individual-based one, in which the stochastic aspects of the dynamics are fully accounted for. As expected, at the IBM level of description the dynamics of the population is richer. In particular, the MA is influencing in a highly nontrivial manner the critical properties of the system at the phase transition point between "alive" and "extinct". A spectacular effect in the phase diagram of the system in the plane of the parameters $\mathcal{S}$ $\mathcal{M}$ (for fixed values of the period and amplitude of the environmental changes) is the existence of an optimal MA, i.e., a value of $\mathcal{M}$ for which the critical SP is maximal. The existence of this optimal MA, that is specific to each intermediate value of $T_{p}$, is strongly reminiscent of the stochastic resonance phenomenon encountered in stochastic systems submitted to a periodic deterministic perturbation, see e.g. [34]. In a broad sense, this effect means that the response of a nonlinear dynamical system to a periodic perturbation of one of its parameters can be enhanced by an optimal amount of noisy stimulation of the system. In our case, the response of the system is encoded in the MET, and the noisy ingredient is the random mutation of amplitude $\mathcal{M}$. A more explicit, quantitative mapping of our system on a nonlinear stochastic equation for the concentration that exhibits the stochastic resonance phenomenon is currently under study.

We also compared the effects of a smooth variation of the optimum with those corresponding to an abrupt changing of the environment (as, for example, a largescale catastrophic event). We showed that sufficiently large mutations can increase dramatically the survival chances of the population in case of a catastrophe.

Variations of the model introduced above, for example using different expressions for the individual fitness, or for the dependence of the probability of extinction upon the SP, and fitting better some possible experimental data, could also be considered. Also, more complicated than diffusive types of motion, that may pertain to a "strategy" of the individuals (e.g., a tendency to approach or, on the contrary, to avoid other individuals) are currently under study. We believe however that most of the qualitative features of our model, resulting from the competition of several basic processes, are generic. Extensions to ecosystems composed of several competing species and submitted to a changing environment is presently under investigation.

\section{Acknowledgments}

The authors thank an anonymous referee for very useful suggestions. M.D. and I.B. acknowledge partial sup- 
port from the Swiss National Science Foundation. M. D. and J. S. acknowledge the COST10-SER-No.C06.0027 program for support. A.P. is grateful for the hospital- ity of the Theoretical Physics group of the University of Geneva.
[1] A. J. Lotka, Proc. Nat. Acad. Sci. USA 6, 410 (1920).

[2] V. Volterra, Leçons sur la théorie mathématique de la lutte pour la vie, Gauthier-Villars, Paris (1931).

[3] F. Schweizer, Modeling Complexity in Economic and Social Systems, World Scientific, Singapour (2002).

[4] C. K. Hemelrijk (ed.), Self-Organisation and Evolution of Social Systems, Cambridge University Press, Cambridge (2005).

[5] M. Droz, in Modeling Cooperative Behavior in the Social Sciences, P. L. Garrido, J. Marro and M. A. Munoz (eds.), AIP Conf. Proc. Vol. 779, Melville, New York (2005).

[6] A. T. Bradshaw and L. L. Moseley, Phys. A 261, 107 (1998).

[7] R. Durrett and S. Levin, Theor. Popul. Bio. 46, 363 (1994).

[8] S. M. Henson et al, Science 294, 602 (2001).

[9] G. Domokos and I. Scheuring, J. Theor. Biol. 227, 535 (2004).

[10] J. B. Mitton, Selection in natural populations, Oxford University Press, Oxford (1997).

[11] M. F. He, Z. Wang, Q. Pan, and Y. Cai, Int. J. Mod. Phys. C 16, 177 (2005).

[12] D. Stauffer, S. Moss de Oliveira, P. M. C. de Oliveira, and J. S. San Martin, Biology, Sociology, Geology by Computational Physicists, Elsevier, Amsterdam (2006).

[13] M. Droz and A. Pȩkalski, Physica A 298, 545 (2001).

[14] M. Droz, F. Coppex, and A. Lipowski, Phys. Rev. E 69, 061901 (2004).

[15] A. Pȩkalski and M. Droz, Phys. Rev. E 73, 021913 (2006).

[16] R. S. Bradley, Quarternary Paleoclimatology, Chapman \& Hall, London (1994).

[17] C. M. Pease, R. Lande, and J. J. Bull, Ecology 70, 1657 (1989).

[18] R. Lande, Am. Nat. 142, 911 (1993).

[19] K. Sznajd-Weron and A. Pȩkalski, Physica A 269, 527 (1999).

[20] R. Gomulkiewicz and R. D. Holt, Evolution 49, 151 (1995).

[21] R. Bürger and M. Lynch, Evolution 49, 201 (1995).

[22] A. Fraser and D. Burnell, Computer Models in Genetics, McGraw-Hill, New York (1970).

[23] M. Droz and A. Pȩkalski, Phys. Rev. E 65, 051911 (2002).
[24] K. Pawlikowski and A. Pȩkalski, Physica A 342, 597 (2004). between

[25] M. Droz and A. Pȩkalski, Phys. Rev. E 69, 051912 (2004).

[26] G. Nicolis, Introduction to Nonlinear Science, Cambridge University Press, Cambridge (1995).

[27] For a pedagogical discussion of finite-size effects in the context of several out-of-equilibrium phase transitions, see R. Toral and C. J. Tessone, Commun. Comput. Phys. 2, 177 (2007).

[28] A. Pȩkalski, Physica A 314, 114 (2002).

[29] M. L. Shaffer, BioScience 31, 131 (1981).

[30] M. E. Gilpin and M. E. Soulé, in Conservation Biology, M. E. Soulé (ed.), Sinauer Associates, Sutherland (1986).

[31] K. Sznajd-Weron, Eur. Phys. J. B 16, 183 (2000).

[32] M. Zawierta, P. Biecek, W. Waga, and S. Cebrat, Theory in Biosc. (2007), in print.

[33] O. Diekmann in Mathematical Modeling of Population Dynamics, Banach Center Publications, Vol. 63, 47 (2004).

[34] R. Benzi, "Stochastic Resonance: from climate to biology", arXiv:nlin.CD/0702008 L. Gaimmaitoni, P. Hänggi, P. Jung, and F. Marchesoni, Rev. Mod. Phys. 70, 223 (1996).

[35] Although hermaphrodite, the individuals need mating for reproduction.

[36] In the biological literature parameters analogous to $\mathcal{M}$ are often referred to as mutation rate. However, from the point of view of the physical aspect it designates, the term mutation amplitude looks more appropriate to us.

[37] One should notice that changing one or both of the values of $\varphi$ and $\langle z\rangle$ is simply equivalent to a rescaling of the selection parameter $\mathcal{S}$ in Eq. (5).

[38] In view of the one-to-one relationship (14) between $P(z, t)$ and $\Pi(p, t)$, one can, alternatively, focus on the probability distribution function of the individual extinction probability, $\Pi(p, t)$.

[39] Adaptive dynamics (see e.g. [33] for an introduction) studies how a population of agents having all the same trait reacts to the presence of an individual (a "mutant") having a different trait. The associated dynamics is described by mean-field like partial differential equations. However, in our case, the generic situation is not of this type, as the traits of the agents are randomly distributed. 